\title{
在大鼠玻璃体内移植猕猴神经前体细胞的方法探索
}

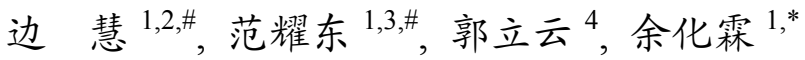

(1. 昆明医学院 第一附属医院微创神经外科, 云南 昆明 650032; 2. 昆明医学院 生理教研室 云南 昆明 650031;

3. 昆明医学院 第三附属医院神经外科, 云南 昆明 650106; 4. 昆明医学院 第四附属医院眼科, 云南 昆明 650021)

摘要: 探索一种简单、可行的异种神经前体细胞眼内移植方法。采用机械性损伤方法造成大鼠视网膜局部受 损，然后在损伤眼及对照眼玻璃体内移植绿色荧光蛋白(green fluorescence protein, GFP)标记的狝猴神经前体细胞， 观察细胞能否存活。结果显示: 经激光共聚焦显微镜检查发现移植细胞在损伤眼及对照眼内均可存活并整合至损 伤眼视网膜。实验表明, 玻璃体内异种移植 GFP 标记的猕猴神经前体细胞可以存活并整合, 是一种可行的移植 方法。

关键词: 猕猴; 神经前体细胞; 玻璃体; 移植

中图分类号：Q959.848; R779.65; Q813.3 文献标志码：A＼cjkstart文章编号：0254-5853-(2012)01-0085-04

\section{A technique of rhesus monkey neural progenitor cells intravitreal transplant to rats}

\author{
BIAN Hui ${ }^{1,2, \#}$, FAN Yao-Dong ${ }^{1,3, \#}$, GUO Li-Yun ${ }^{4}$, YU Hua-Lin ${ }^{1, *}$ \\ (1. Department of Minimally Invasive Neurosurgery, First Affiliated Hospital of Kunming Medical College, Kunming 650032,China; 2. Department of \\ Physiology, Kunming Medical College, Kunming Yunnan 650031, China; 3. Department of Neurosurgery, Third Affiliated Hospital of Kunming Medical \\ College, Kunming Yunnan 650106,China; 4. Department of Ophthalmology, Fourth Affiliated Hospital of Kunming Medical College, Kunming 650021,China)
}

\begin{abstract}
To investigate a simple and effective intraocular xenotransplant technique of rhesus monkey neural progenitor cells to rats, mechanical injury was induced in the rat's right retina. And the GFP-labeled rhesus monkey neural progenitor cells suspension was slowly injected into the vitreous space of the right injured and left control eye. Confocal image suggested that the xenografted cells survived in both the injured and control eye, meanwhile the cells integrated in the injured right retina. The results demonstrated that intravitreal xenotransplant could be adopted as a simple and reliable method.
\end{abstract}

Key words: Rhesus monkey; Neural progenitor cells; Vitreous; Transplant

玻璃体是一种高度水化的细胞外基质成分，呈 无色透明凝胶状态, 主要由水 $(>95 \%)$ 、胶原纤维、 透明质酸和可溶性蛋白组成。此外, 还含有白蛋 白、硫酸软骨素、葡萄糖、氨基酸、脂肪酸及无机 离子等成分, $\mathrm{pH}$ 值接近中性, 构成眼内最大容积 (Bishop, 2000; Kleinber et al, 2011 )。玻璃体的胶冻 状结构是一种理想的培养基, 可以给细胞提供一个 良好的眼内代谢微环境, 为移植细胞的长期存活及
广泛迁移提供营养支持(Coles et al, 2004)。以往的研 究显示, 将成年大鼠海马来源的神经前体细胞移植 至成年大鼠玻璃体腔内, 移植细胞至少可以存活 8 周 (Takahashi et al，1998); 增强型绿色荧光蛋白 (enhanced green fluorescence protein, EGFP)标记的 转基因小鼠脊髓来源的神经前体细胞细胞移植到 成年小鼠玻璃体内可以存活 6 个月(Pressmar et al, 2001)。

\footnotetext{
收稿日期: 2011-10-27; 接受日期: 2011-12-30

基金项目: “973” 计划 (2007CB947703)

*通信作者(Corresponding author), E-mail: yuh1308@126.com

\#并列第一作者(Authors contributed equally to the work)

第一作者简介: 边 慧, 昆明医学院在读博士研究生, E-mail: bh_032001@yahoo.com.cn
} 
视网膜由胚胎时期神经外胚层形成的视杯发 育而来, 是高度特化的神经组织。作为中枢神经系 统的外延部分, 视网膜组织结构相对简单, 细胞呈 清晰而明显的分层状排列(Klassen et al, 2004)。视网 膜在神经干细胞移植研究中具有独特的部位优势。 由于眼底可以直视, 当以 GFP 标记的细胞作为移植 供体时，应用眼底苂光照相或长波紫外线可以对 GFP 发出的菼光直接进行活体重复观察, 即可通过 非侵入性的方法获取和追踪移植细胞的存活、迁移 及与宿主的联系等情况(Bennett et al, 1997)。此部位 实验操作、取材方便, 不需要进入脑内观察供、受 体神经细胞之间的联系及整合, 且充分体现出外延 的优势, 因此, 在干细胞移植研究中得到广泛的应 用。

狝猴在进化上是与人类非常相近的动物，其生 理学、解剖学、遗传学、系统发育学等方面与人类 非常相似(Calhoun et al, 2003; Kuai et al, 2009), 有 关非人灵长类神经前体细胞的研究不仅可以加深 对干细胞生物学的基础理论理解, 而且对实现干细 胞替代治疗具有重要意义。 Li et al（2005）前期的 研究工作表明, 将猕猴胚胎干细胞诱导分化而来的 神经前体细胞异种移植到成年大鼠脑内, 移植细胞 能够在受体脑内存活并且分化为神经元和神经胶 质细胞。猕猴神经前体细胞作为替代治疗的上选细 胞株能否异种移植至啮齿类动物玻璃体，目前尚未 见相关报道。近年来的研究表明, 受损伤的视网膜 可以易化眼内移植局部微环境, 有利于移植细胞的 存活及整合(Bull et al, 2008; Young et al, 2000)。为此, 本研究采用机械性损伤方法建立大鼠视网膜局部 损伤模型, 损伤后将 GFP 标记的猕猴神经前体细胞 异种移植入玻璃体，观察细胞在玻璃体内能否存活， 从而探索一种可行的移植方式, 为细胞替代治疗视 网膜疾病提供实验基础。

\section{1 材料与方法}

\section{1 实验动物及分组}

成年 SPF 级雄性 Sprague-Dawley 大鼠, 体重 $180 \sim 200 \mathrm{~g}$, 购于昆明医学院实验动物中心, 许可 证编号 SCXK(滇)2011-0004。所有实验用鼠自购买 后, 在本实验室至少适应一周。大鼠饲养在动物笼 内, 自由进水、取食, 12 小时明: 暗交替, 室温(23 $\pm 2){ }^{\circ} \mathrm{C}$, 操作符合动物实验管理条例。大鼠分为 2 组: 损伤组(右眼) $(n=25)$; 对照组(左眼) $(n=25)$ 。

\section{2 模型建立及细胞移植}

硫酸阿托品注射液(天津金耀氨基酸有限公司) $0.3 \mathrm{~mL} /$ 只, $10 \%$ 水合氯醛(国药集团化学试剂有限公 司) $400 \mathrm{mg} / \mathrm{kg}$ 体重腹腔注射致动物麻醉后，将大鼠 固定在脑立体定位仪上(深圳瑞沃德生命科技有限 公司)。碘伏消毒结膜囊及睑裂周围皮肤, 结膜囊内 滴贝诺喜眼液(Alcon Laboratories, Inc) 1 滴行表面 麻醉, 复方托吡卡胺滴眼液(Alcon Laboratories, Inc) 1 滴散大瞳孔。GFP 标记的狝猴神经前体细胞由中 科院昆明动物所王正波博士惠赠 (GFP 标记的狝猴 肧胎干细胞团块悬浮培养，形成的胚体贴壁培养， 诱导分化形成具有玫瑰花环结构的神经前体细胞, 备用)。用 $5 \mu \mathrm{L}$ 的微量进样器吸取 $3 \mu \mathrm{L}$ (约含细胞 1 $\times 10^{5}$ 个 $\left./ \mu \mathrm{L}\right) \mathrm{GFP}$ 标记的狝猴神经前体细胞悬液备 用。眼科镊提起球结膜, 暴露大鼠角巩膜缘, 在其 后约 $4 \mathrm{~mm}$ 处 12:00 位置进针。注意保持与地面垂 直的角度，继续进针到玻璃体内，将对侧 6:00 处视 网膜划伤，造成视网膜机械性损伤，具体实验模型 制作方法参照文献(Nisbida et al, 2000)进行。稍退针 至玻璃体内注射猕猴神经前体细胞悬液 $3 \mu \mathrm{L}$ 。手术 结束时, 结膜囊内涂红霉素眼药膏预防感染。术后 继续采用预防感染措施 $3 \mathrm{~d}$, 白天滴氯霉素眼药水, 晚上涂红霉素眼药亳。

\section{3 移植后处理}

移植细胞后 7 周, 将大鼠深度麻醉后, 经左心 室插管分别使用 $0.9 \%$ 氯化钠溶液和 $4 \%$ 多聚甲醛溶 液灌注固定, 仔细分离出鼠左右眼球后, 将其固定 于 $4 \%$ 多聚甲醛溶液，置于 $4{ }^{\circ} \mathrm{C}$ 冰箱过夜保存。经 $20 \% 、 30 \%$ PBS 蔗糖溶液梯度脱水后, 使用冰冻切 片机(LEICA CMI850)切片, 片厚 $20 \mu \mathrm{m}$ 。激光共聚 焦显微镜 (ZEISS LSM 510 META)观察移植细胞存 活及分布情况。

\section{2 结 果}

\section{1 动物眼球观察}

猕猴神经前体细胞移植后，观察大鼠双侧眼球， 可见角膜透明，玻璃体腔内可见点状、条索状或网 状灰白色混浊物, 各组均未见眼内感染。

\section{2 组织学检查}

切片经 HE 染色显示, 右眼视网膜由于机械性 损伤造成视网膜结构紊乱，结构层次被破坏，神经 上皮层扭曲，断裂，部分细胞萎缩消失。损伤灶周 围可见成簇红细胞(图 1)。 


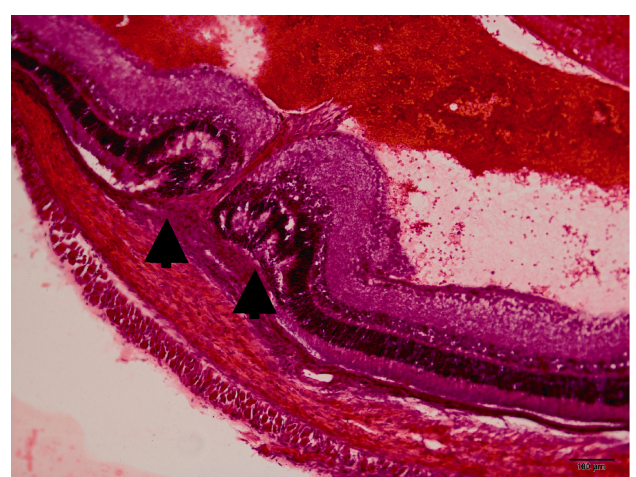

图 1 机械性损伤大鼠右眼局部视网膜

Fig.1 Mechanical injury in the right retina of rat $\mathrm{HE}$ 染色法, $\times 100$ 。箭头示视网膜结构破坏, 细胞排列紊乱。 Hematoxylin-Eosin staining, $\times 100$ )(Arrow indicates the retinal structure was disordered and the cells of the injured sites are in disarrangement.

\section{3 细胞移植后观察}

冰冻切片后，在激光共聚焦显微镜下观察发现，
对照眼(左眼)玻璃体内有大量移植 GFP 阳性细胞存 活(图 2), 损伤眼(右眼)局部视网膜上出现 GFP 阳性 细胞整合(图 3)。

\section{3 讨 论}

判断局部微环境是否适合移植细胞生存以及 移植方法是否切实可行是移植工作的重要组成部 分。在移植工作中要尽量做到对宿主组织、供体细 胞或组织自身损伤小，从而确保移植细胞的有效存 活，因此，选择更适合、更可靠、更行之有效的异 种神经前体细胞移植方法仍需探索 (Lund et al, 2001)。本研究采用机械性损伤方法造成大鼠右眼视 网膜局部损伤后，在玻璃体内移植细胞观察存活情 况。与此同时，以正常左眼作为对照亦系移植细胞， 观察神经前体细胞在两者中的存活情况。结果发现, 无论在损伤眼还是对照眼内狝猴神经前体细胞均 可存活。该结果与 Grozdanic et al (2006)研究相似。
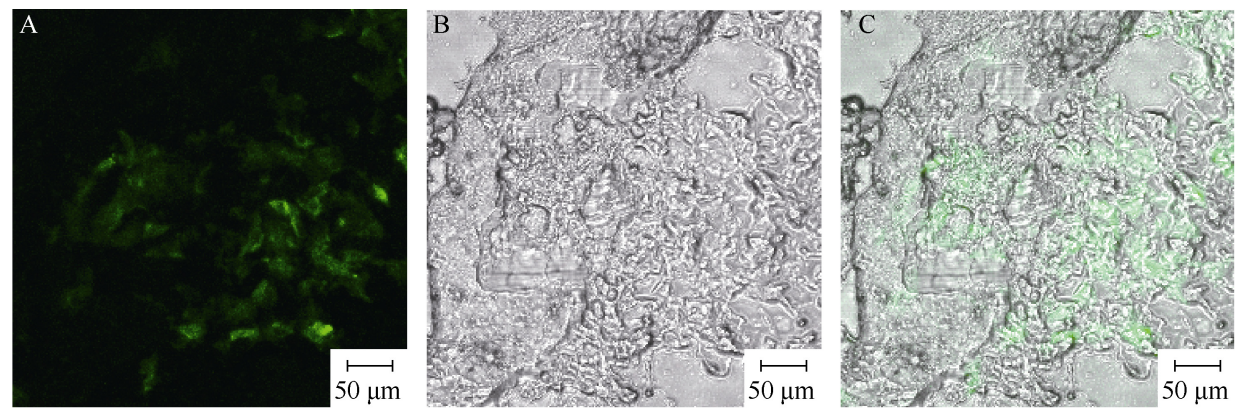

图 $2 \mathrm{GFP}$ 标记的猕猴神经前体细胞移植至大鼠对照眼玻璃体内

Fig.2 GFP positive rhesus monkey neural progenitor cells xenotransplant in the left eye of rat A）GFP 阳性细胞；B）对照眼玻璃体；C）A、B叠加图。

A) GFP positive cells; B) vitreous space C) A \& B merged image.
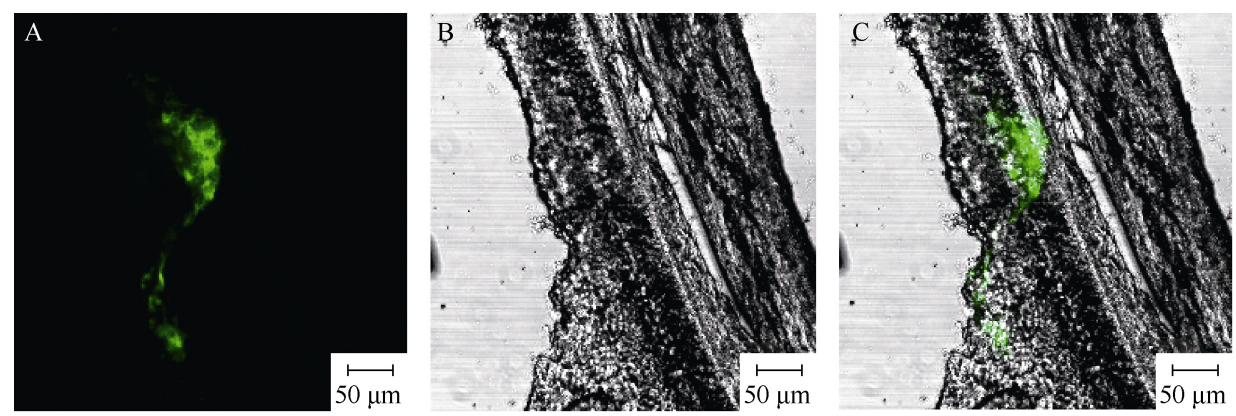

图 3 GFP 标记的猕猴神经前体细胞整合至大鼠损伤眼视网膜

Fig.3 GFP positive rhesus monkey neural progenitor cells integrated in the injured retina of the rat A) GFP 阳性细胞；B）损伤眼视网膜；C）A、B 叠加图。

A) GFP positive cells; B) retina of the right eye; C) A \& B merged image.

目前常用的眼内移植干细胞的方法有经玻璃 体和经巩膜 2 种途径。经巩膜通路(视网膜下移植)
对技术及设备要求相对更高且操作复杂, 手术时, 术野暴露有限，会破坏脉络膜血管和 Bruch's 膜，导 
致视网膜内外层之间分离以及血眼屏障破坏、受体 早期致敏等并发症(Al-Amro et al, 1999; Lund et al, 2001)。经玻璃体通路术野清晰, 容易操作, 可控制 性更好, 损伤相对小, 并不破坏视网膜的屏障解剖 结构, 而且玻璃体可以为移植细胞长期存活和广泛 迁移提供营养支持, 故经玻璃体通路更具优势(Baker \& Brown, 2009; Coles et al, 2004; Meyer et al, 2004)。 本研究采用经玻璃体通路法进行移植, 手术过程中 通过散大的睲孔可以清晰看到微量注射器针头的 走向及注射部位。这种近似直视下的操作对移植靶 组织造成的损伤相对更小, 且感染发生率低(本实验 无一只眼球术后出现感染)。移植后方便观察, 可同 时进行双侧对比, 透过睲孔可见白色点状物和絮状 物在玻璃体内生长。本研究采用机械性损伤方法造 成大鼠视网膜受损后, 在损伤眼及正常眼内经玻璃 体通路异种移植 GFP 标记的猕猴神经前体细胞，观 察细胞存活情况。结果显示 GFP 标记的猕猴神经前 体细胞在玻璃体腔内至少能存活 7 周, 表明经玻璃 体异种移植猕猴神经前体细胞是一种可行的移植方 式。本研究为探索更佳的动物模型建立方法及可行 的细胞移植方法奠定了基础, 为进一步探索视网膜

\section{参考文献:}

A1-Amro S, Tang L, Kaplan HJ. 1999. Limitations in the study of immune privilege in the subretinal space of the rodent[J]. Invest Ophth Vis Sci, 40(12): 3067-3069.

Baker PS, Brown GC. 2009. Stem-cell therapy in retinal disease[J]. Curr Opin Ophthalmol, 20(3): 175-181.

Bennett J, Duan DS, Engelhardt JF, Maguire AM. 1997. Real-time, noninvasive in vivo assessment of adeno-associated virus-mediated retinal transduction[J]. Invest Ophthalmol Vis Sci, 38(13): 2857-2863.

Bishop PN. 2000. Structural macromolecules and supramolecular organisation of the vitreous gel[J]. Prog Retin Eye Res, 19(3): 323-344.

Bull ND, Limb GA, Martin KR. 2008. Human müller stem cell (MIO-M1) transplantation in a rat model of glaucoma: survival, differentiation, and integration[J]. Invest Ophth Vis Sci, 49(8): 3449-3456.

Calhoun JD, Lambert NA, Mitalipova MM, Noggle SA, Lyons I, Condie BG, Stice SL. 2003. Differentiation of rhesus embryonic stem cells to neural progenitors and neurons[J]. Biochem Biophys Res Commun, 306(1): 191-197.

Coles BLK, Angénieus B, Inoue T, Del Rio-Tsonis K, Spence JR, Mclnnes RR, Arsenijevic Y, van der Kooy D. 2004. Facile isolation and the characterization of human retinal stem cells[J]. Proc Natl Acad Sci USA, 101(44): 15772-15777.

Grozdanic SD, Ast AM, Lazic T, Kwon YH, Kardon RH, Sonea IM, Sakaguchi DS. 2006. Morphological integration and functional assessment of transplanted neural progenitor cells in healthy and acute ischemic rat eyes[J]. Exp Eye Rea, 82(4): 597-607.

Klassen H, Sakaguchi DS, Young MJ. 2004. Stem cells and retinal repair[J]. Prog Retin Eye Res, 23(2): 149-181.

Kleinber TT, Tzekov RT, Stein L, Ravi N, Kaushal S. 2011. Vitreous substitutes: a comprehensive review[J]. Surv Ophthalmol, 56(4): 300-323.
疾病的干细胞移植研究和治疗提供了技术支持。

另外，前人的工作提示，成年大鼠海马源性神 经前体细胞移植至成年大鼠玻璃体后可形成一个 紧邻视网膜神经纤维层的连续的细胞薄层, 该薄层 主要生长在靠近内界膜处, 其间有少量移植细胞可 整合到宿主视网膜，但并不破坏宿主的视网膜结构 (Takahashi et al, 1998), 将胚胎小鼠脊髓源性神经前 体细胞移植到成年野生型小鼠及视网膜营养不良 突变型小鼠玻璃体内, 无论是正常成年小鼠的视网 膜还是营养不良的视网膜内, 异位移植的细胞均可 存活、分化及整合。移植细胞胞体多位于视网膜的 内网状层, 细胞突起可伸入神经纤维层(Pressmar et al, 2001)。目前本实验的工作只是初步证明了 GFP 标记的猕猴神经前体细胞能够在大鼠玻璃体内存 活，并发现少量移植细胞可整合至宿主视网膜。移 植细胞能否在形态上向视网膜样细胞分化, 以及是 否具有视网膜细胞功能还需进一步进行研究。

致谢: 感谢中科院昆明动物所马原野研究员、 胡新天研究员给予的帮助，感谢王正波博士给予 的帮助。

Kuai XL, Gagliardi C, Flaat M, Bunnell BA. 2009. Differentiation of nonhuman primate embryonic stem cells along neural lineages[J]. Differentiation, 77(3): 229-238.

Li TQ, Zheng JW, Xie YH, Wang SF, Zhang XZ, Li J, Jin LF, Ma YY, Wolf DP, Zhou Q, Ji WZ. 2005. Transplantable neural progenitor populations derived from rhesus monkey embryonic stem cells[J]. Stem Cells, 23(9): 1295-1303.

Lund RD, Kwan AS, Keegan DJ, Sauvé Y, Coffey PJ, Lawrence JM. 2001. Cell transplantation as a treatment for retinal disease[J]. Prog Retin Eye Res, 20(4): 415-449.

Meyer JS, Katz ML, Maruniak JA, Kirk MD. 2004. Neural differentiation of mouse embryonic stem cells in vitro and after transplantation into eyes of mutant mice with rapid retinal degeneration[J]. Brain Res, 1014(1-2): 131-144.

Nisbida A, Takabashi M, Tanibara H, Nakano I, Takabashi JB, Mizoguchi A, Ide C, Honda Y. 2000. Incorporation and differentiation of hippocampus-derived neural stem cells transplanted in injured adult rat retina[J]. Invest Ophth Vis Sci, 41(13): 4268-4274.

Pressmar S, Ader M, Ricbard G, Scbacbner M, Bartsch U. 2001. The fate of heterotopically grafted neural precursor cells in the normal and dystrophic adult mouse retina[J]. Invest Ophth Vis Sci, 42(13): 3311-3319.

Takahashi M, Palmer TD, Takahashi J, Gage FH. 1998. Widespread integration and survival of adult-derived neural progenitor cells in the developing optic retina[J]. Mol Cell Neurosci, 12(16): 340-348.

Young MJ, Ray J, Whiteley SJO, Klassen H, Gage FH. 2000. Neuronal differentiation and morphological integration of hippocampal progenitor cells transplanted to the retina of immature and mature dystrophic rats[J]. Mol Cell Neurosci, 16(3): 197-205. 